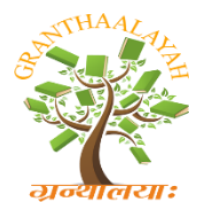

\author{
INTERNATIONAL JOURNAL OF RE
GRANTHAALAYAH \\ A knowledge Repository
}

Science

\title{
DEMONSTRATION OF PRECISE RELATIONSHIPS AND LINKS BETWEEN TYPE 2 DIABETES MELLITUS, INSULIN RESISTANCE AND PARKINSON'S DISEASE
}

\author{
Habib ur Rehman ${ }^{1}$, Kaleemullah ${ }^{1}$, Abdul Malik Tareen ${ }^{1}$ \\ ${ }^{1}$ Department of Microbiology, University of Balochistan Quetta Pakistan
}

\begin{abstract}
Diabetes is a metabolic disorder that intessssrferes with the body's ability to consume food and convert it into energy. The most common mode of diabetes in type 2 diabetes mellitus (T2DM) is that the body cannot effectively use insulin produced by the pancreas. This is called insulin resistance. Parkinson's Disease (PD) is a chronic neurodegenerative motor defect whose properties work is hindrance with locomotion. This is due to the loss of neurons in the substantia nigra area under the brain that produces dopamine, a chemical messenger that transmits signals that produce smooth, meaningful movement. Dopamine loss caused by neuronal damage and death causes impaired movement. Cells rely on glucose for energy, and insulin helps to convert glucose into energy. In both Parkinson's disease and type 2 diabetes, changes in the brain can result in signaling interruptions that affect normal functioning. Insulin resistance also reaches the brain and produces severe changes in the nerve cells, increasing the risk of Parkinson's disease and abnormally promoting high blood sugar leads to high-rise the inflammation, associated with the development of Parkinson's disease. Diabetes suffering peoples are more likely to have Parkinson's disease. Parkinson's disease is $32 \%$ higher in people with type 2 diabetes than in non-diabetic patients. Patients with type 2 diabetes along with its complication have a $49 \%$ higher risk of Parkinson's disease, while those having only type 2 diabetes without complications have a $30 \%$ risk of parkinson's disease. In particular, younger patients with diabetes (25 to 44 years of age) have an approximately fourfold increased risk of developing Parkinson's compared with adults of similar age without diabetes. Researchers has estimated the high risk of developing PD based on longitudinal data methodology for people with type 2 diabetes. Initial studies have reported more than 400 genes linked in both conditions. The potential link between type 2 diabetes and Parkinson's disease has been the topic of medical dialogue and scientific research for years. Recently, animal and in vitro studies have shown that the pathophysiology and clinical symptoms of Parkinson's disease are concerned more with insulin dysregulation and changes in insulin action.
\end{abstract}

Keywords: Type 2 Diabetes Mellitus; Insulin Resistance; Parkinson:s Disease.

Cite This Article: Habib ur Rehman, Kaleemullah, and Abdul Malik Tareen. (2019). "DEMONSTRATION OF PRECISE RELATIONSHIPS AND LINKS BETWEEN TYPE 2 DIABETES MELLITUS, INSULIN RESISTANCE AND PARKINSON'S DISEASE." 
International Journal of Research - Granthaalayah, 7(12), 259-270. https:// doi.org/10.29121/granthaalayah.v7.i12.2019.320.

\section{Introduction}

Human behavior and lifestyle changes have increased the prevalence of diabetes mellitus (DM) globally, and it is estimated that 422 million people with DM have been reported already in 2014. The global number of people with DM is estimated to be 642 million affected by 2040 (IDF, 2015). Type 2 diabetes is a disease in which blood glucose levels are high to limits. Insulin is a hormone in the body that helps to move glucose into the cells for energy. In the people with type 2 diabetes, the body does not use insulin properly so also known as insulin resistance. Type 2 diabetes is one of the fastest developing health problems globally, and it is linked with a different kind of complications. Over time, high blood glucose levels cause serious problems, damage to eyes, kidneys and nerves, and increase the risk of heart disease, stroke and organ dysfunction like loss of limbs due to amputation. 1Epidemiological studies have indicated the coronary heart disease more risk in type 2 Diabetes as compared to non-diabetes sufferers. Evidence from prospective epidemiological studies for a possible pandemic disease showed that type 2 diabetes triggers hyperglycemia condition for multiple linked hyperglycemia complication virtually in all the organs, including neurodegenerative diseases such as diabetic neuropathy, stroke, dementia, Alzheimer's disease, and the high risk of Parkinson's disease (IDF \& WHO, 2015). It is generally proved that Parkinson's disease is caused by destruction of brain cells that produces a molecule called dopamine. Dopamine is one of the molecules used by the nerve cells in the brain to communicate with one another. Lack of this molecule leads to difficulties with movement. About half of those also suffer hallucinations and delusions. Parkinson disease (PD) is a progressive neurodegenerative disease (Schapira, 2008). The pathogenesis of Parkinson's disease is gradually being explained by the contribution of overlapping and interconnected pathways, including mitochondrial turnover (mitophagy and mitochondrial biogenesis), neuroinflammation and accumulation, and disaggregation of toxic heterogeneous protein. Significant stages in this neurodegenerative pathway have been explored emphasizing the linkages between the Parkinson's disease pathogenesis and progressive developing of insulin resistance mechanisms. This is particularly noteworthy because many agents used in the treatment of type 2 diabetes have been shown to be neuroprotective in animal models of Parkinson's disease and are now being tested in randomized controlled testing trials in Parkinson's disease patients (Belsham et al., 2009).

\section{Associations Between Type 2 Diabetes and Parkinson's Disease: Type 2 Diabetes Rises the Threat of Parkinson's Disease (PD)}

On the basis of greater risk of the prevalence of PD in diabetic patients, the pervasiveness of diabetes in the people of PD is higher than that of non-PD individuals. It is estimated that $8-30 \%$ of the people with PD have diabetes. Additionally, 50-80\% of people with PD have abnormal glucose tolerance tests. Diabetes appears as to accelerate the development of both motor and nonmotor symptoms of PD (Schapira, 2008; Kwak et al,. 2010).

Association concerning with type 2 diabetes mellitus (T2DM) and neuro-degeneration has been recommended for decades. Numbers of research studies have considered at the association among Parkinson's disease (PD) and type 2 diabetes (Craft, 2004). Oxidative stress, inflammation and insulin insufficiency or resistance has been suggested as a common threat features for PD, 
Alzheimer disease (AD), as well as for T2DM. Both DM and PD (or AD) is neurodegenerative protein conformational disorders. Alzheimer disease (AD) has even been measured as a brain particular type of diabetes considered by insulin resistance, a "Type 3 Diabetes" (Sun et al., 2014; Jin et al., 2012).

Proof from prospective epidemiological studies has shown the type 2 diabetes mellitus as an independent risk disorder has led to many complications of nervous system linked diseases such as diabetes neuropathy (Boulton et al., 2005), stroke (Tuomilehto et al., 1996; Hu et al., 2007), and more recently, the Alzheimer's disease (Leibson et al., 1997; Ott et al., 1999; Peila et al., 2002). There have also been reports the different association from various cross-sectional and cohort studies on different fields between diabetes or abnormal glucose tolerance and the sporadic forms of Parkinson's disease. Survey data shows that diabetes accounts for 8 to 30 percent of Parkinson's disease affected patients, consistently in excess of the prevalence found in non-Parkinson's disease individuals (Chalmanov and Vurbanova, 1987; Pressley et al., 2003). High prevalence of Parkinson's disease with increased hyperglycemia can easily be explained on the basis of regular attending or hospitalizations by additional medical contact / urine / blood tests. The association reinforced the report by suggesting that $50-80 \%$ of Parkinson's patients have abnormal glucose tolerance during the test (although these data are non-synchronous and that we are unaware to used validated data from recent cohorts (Barbeau et al., 1961; Elner and Kandel, 1965; Lipman et al.,1974; Sandyk, 1993); however, in a series of 800 patients with Parkinson's disease, diabetes has shown actually a strong association to accelerate the development of both motor and Cognitive symptoms (Schwab, 1960). In the view of the given potential complex effects of Parkinson's disease treatment, it has never been studied the adults with Parkinson's disease and been shown to reduce the amount of insulin-mediated glucose uptakes in adults with Parkinson's disease (Van Woert and Mueller,1971 inhibition of early insulin secretion and long-term hyperinsulinaemia and hyperglycaemia after glucose loading (Boyd et al.,1971).

Concerning with correlation among DM and PD and on the basis of exploration of epidemiological cohort studies, the diabetes has advocated as a threat element for future PD, and extensive connection between the hereditary risks associated with both diseases has also been described. In addition, the common pathophysiological mechanisms in both DM and PD conditions via preclinical in-vitro and in-vivo studies has also been implicated. Patients with DM having more aggressive form of PD are more axial symptoms than without DM, better metabolic control of DM while arising in PD (Huang et al., 2015).

Current researches emphasized the possible association among Diabetes Mellitus (a very common condition) and Parkinson's disease (PD). There is strong need of good quality epidemiology for exploring the possible relationship between PD and Diabetes. According to Foltynie et al., 2006, circumstantial observations from treatment centers of Diabetes or PD has confirmed the indeed presence of certain patients with both disorders. As a rule, cohort studies include more participants and are more truly with lower probability of bias than case control studies. In cohort studies, there is always certainty about diabetes that it is consistently a threat feature for future PD. While in the case-control studies, there was no relationship among diabetes and PD (Yue et al., 2016).

While according to Politis et al, (2006), in seven meta-analysis observational cohort studies (regarding prospective or retrospective) considered the linkages of earlier-exist type 2 diabetes 
with the risk of emerging PD in over 1.7 million individuals. Patients with type 2 diabetes had a $38 \%$ higher risk for emerging PD compared with nondiabetic patients. This result remain unchanged depending upon the study area, study value, research nation, study proposal and sample size, issued year and sensitivity evaluates, which confirmed the strength of the diseases linkages. On the basis of both observations, firstly it is suggested that patients with type 2 diabetes might have a high threat for developing PD, secondly though the prevalence of diabetes is not greater in patients with PD compared to the common population.

Another facilitating method for the diagnosis of coordination between DM and PD researchers, suggested the role of insulin regulation pathway and explained the role of insulin in the regulation of brain dopaminergic activity and disregulation of insulin has contributed to PD via disease specific or general methods has been presented. The exposure of brain functions via image study also reported that insulin resistance increased in the brain tissue of PD patients as compared to the normal controlled group, and further suggested that a strong mechanistic relationship between insulin resistance and brain structure in PD patients (Morris et al., 2014).

British research suggested the adults with diabetes who has already developed diabetes-related health complications including damage to the retina, kidneys or nerves, faced a 49 percent hike in their Parkinson's risk. Further, men and women with type 2 diabetes may face a significantly higher risk of developing Parkinson's disease later in life (Foltynie et al., 2006).

The association between DM and PD was not consistent with many epidemiological reports with a positive relationship (Lu et al., 2014). Lu et al (2014) conducted a meta-analysis of 14 reports and concluded that the evidence from a case-control study suggests that there may be a lower incidence of PD than a significant heterogeneity. In an another meta-analysis examined this line of questioning, includes cohort and case-control studies, the pooled results of 4 cohort studies with a larger sample size showed that a significant associated risk of diabetes with a significant increased risk of 37\% PD was reported (Saguil and Lax, 2014).

Many authors has used the National Health Insurance Research Database (NHIRD) in Taiwan to study the association of PD and DM, however, none of these studies simultaneously examined the status of diabetes, comorbidity, and medications (Sun et al., 2012; Wahlqvist et al., 2012).

Kaplan-Meier Curve study found that the cumulative incidence statistics of PD was significantly higher in the DM cohort than in non-DM cohort (log-rank test $\mathrm{P}<0.001$ ). Daily analyzes of the natural-grade Cox proportional hazards regression indicated the diagnosis of 1.19-fold increased risk of PD in DM patients as in comparison with non-DM patients after age and comorbidities were kept fixed (Kaplan \& Meier., 1958; Manish et al, 2010).

Yang and his colleagues indicated in their study that the Chinese population is facing an increased risk of PD due to the main cause of DM with a 20 years age and older over 73 years in the followup. The increased rate of association of DM and PD is higher in the women population with age 65 years and older; those without schizophrenia, bipolar disorder, hypertension, high blood pressure, hyperlipidemia, COPD, CAD, stroke, asthma, flunarizine use, CCI score of 0 and number of outpatients percentile visits $\geq 27$; and those using metoclopramide and zolpidem (Yang et al, 2017). 
Dr. Tom Warner of University College London Reported a report to the American Academy of Neurology, describing the "researchers reviewing retrospective study based on the reviewing of hospital admissions statistical data" of more than 8 million people in England over a 12 years period. Investigators were involved in the estimation of risk with the development of PD based on authentic longitudinal data for those with T2DM (Szendroedi et al., 2012). Researchers looked at 2 million people admitted to the hospital for T2D and compared them to a control group of over 6 million people admitted other medical or surgical reasons. Anyone with a PD diagnosis at first admission was excluded from the results, as were those with parkinsonism and other conditions that mimic PD (Warner et al., 2012). Following these cases over the time then through after regular hospital admissions, some basic controls are used in age, gender and residential areas, the authors estimated the $32 \%$ increase of risk (Wiederkehr \& Wollheim, 2006; Zimmet et al., 2001).

In a British study, people with T2D were 32\% more likely to get the chances of Parkinson's disease along with greater risk of later developing the increasingly debilitating neurological disorder at some point in the future than people without diabetes. It appears that the risk is even advanced more than three times the chance for younger people, those between the age 25-44, as well as for those who suffer complications from diabetes (Foltynie et al., 2006). Scientists found that this younger age group is $400 \%$ more likely to develop Parkinson's. A study conducted by several scientists at the University of Oxford and various other study facilities in the United Kingdom has connected Type 2 diabetes with Parkinson's disease. University of London and University of Oxford confirmed the PD 49\% increases in those with medical complications from diabetes, while in the age 75 year old category, the likelihood of take place to develop PD was only $18 \%$ (Wiederkehr \& Wollheim, 2006).

Focusing on the association among the two disorders has been the emphasis of several studies by Dr. Alastair Noyce from Queen Mary's Wolfson Institute of Preventive Medicine. A deeper review of the connection between diabetes and Parkinson's leads researchers to a statement that the two conditions may respond to communal therapies, the goal would be to better understand the association between the two disorders including the influence of genetics, the pathophysiology, and the influence of diabetes on the brain (Alastair et al., 2017; Zimmet et al., 2001).

Few researchers speculated the participants with genes for Type 2 diabetes could also have genes for Parkinson's disease, or some mechanism involved in causing Type 2 diabetes could also cause the added disease (Liao et al., 2015).

In another published study, the 14,252 people with type-2 diabetes were diagnosed with Parkinson's disease during a later hospital admission compared with 20,878 in the comparison group. Researchers determined those with type- 2 diabetes were 32 per cent more likely have a subsequent diagnosis of Parkinson's disease after some exclusions were made including similar conditions, age and gender. Further, the research determined people aged between 25 and 44 had a stronger link between the two conditions (Sun et al., 2012).

Worldwide about 10 million patients live with Parkinson's disease. It is usually diagnosed in people aged 60 or over, but about 4 percent are diagnosed in individuals 50 or younger. It is nearly twice as common in men as in women (Yang et al, 2017). 
American Diabetes Association recommended different techniques to lessen the risk of rising diabetes via healthy lifestyle activities like sustaining a healthy weight, eating healthy and getting regular physical exercise indirectly leads to manage and maintain the health from PD (Jin et al., 2014).

Warner et al, (2012) published their findings from "English Hospital Episode Statistics" database has identified 2 million British patients newly diagnosed with diabetes. The said group was then measured-up against 6 million British patients who had primarily required care during the same time frame for non-diabetes related issues, such as sprains, varicose veins, appendectomies or hip replacements. Over 14,000 of the 2 million in the diabetes group were later diagnosed with Parkinson's matched with about 21,000 of the 6 million others. That translated into a more than 30 percent greater risk for Parkinson's among those with diabetes. Among diabetic patients 25 to 44 years old, 58 years of over 130,700 people established Parkinson's compared with 280 out of nearly 2.6 million similarly aged non-diabetics. That translated into a fourfold greater Parkinson's risk among those with diabetes (Sun et al., 2012).

To prove a cause and effect relationship between type 2 diabetes and Parkinson's, Warner et al, (2012) cited two potential explanations for an association between these two diseases. Firstly there could be "shared genetic predisposition to develop (both) type 2 diabetes and Parkinson's" Secondly there may be "shared pathways in leading to development of diabetes and Parkinson's". While the precise truth of such an association remains indistinct, Warner suggested these diseases connection might engage in the insulin production and glucose control problems that characterize diabetes (Sun et al., 2012). "Unlike most tissues in the body, brain cells are almost totally dependent on glucose as a source of energy". "So if there is a problem in how insulin controls the use of glucose by cells, this may affect certain groups of brain cells selectively lead to Parkinson's." According to Okun et al., (2016), "There are many potential explanations for a link between the Parkinson's and diabetes, but in younger patients, genetics likely plays a key role. In older patients, the degenerative process itself may disrupt brain-driven endocrine pathways, including those related to insulin and to sugar management."

The relationship between type 2 diabetes and Parkinson's is considered to be the most conversation about which may indicate its slow progression therapeutic potential, better treatment in both conditions. According to Dr. Tom Warner, if there is a way to restore the brain's ability to process insulin, there could potentially be a protective effect on the brain (Warner et al., 2012). Okun et al., (2016) studies have described that diabetes treatments have demonstrated potential in relieving Parkinson's Disease symptoms and possibly modifying the course of the disease. Future advanced research base on the examination of insulin signaling, cellular damage, genetic predisposition, and environmental factors will likely be one step forward of detecting and treating in both diabetes and Parkinson's.

\section{Mechanisms involve in the Association of Type 2 Diabetes and Parkinson's Disease.}

According to Foltynie et al, (2006), atherosclerosis of small and large vessel developed in Diabetes having capability to cause vascular Parkinsonism instead of neurodegenerative PD, and spontaneously this may lead to attractive description of the diseases linkages. However; epidemiology suggested that the association $b / w$ the diseases are sustained after elimination of vascular disease from patients. Common genetic risk factors might also be involved in the diseases 
association. The appearance of overlapping linkages regarding mitochondrial dysfunction and inflammatory cascades along with insulin signaling pathways associated with both diseases. Significant correspondence between the genetic threats associated with both diseases has been formally explored using network-based approaches. The perception of insulin signaling/insulin resistance involvement considerably relevant to neuro-degeneration is becoming of growing research. The death of dopaminergic neurons occur due to loss of insulin receptor mRNA in the substantia nigra of PD patients. Additionally, in 50-80\% of PD patients there were insulin resistance detection formally tested for glucose tolerance, but it is uncertain either the central insulin resistance in PD is a cause for or a consequence of neuro-degeneration.

Accumulation of abnormal protein in diabetes and PD also showed further insights of association in many studies. With the similar idea of abnormal propagation of pathological alpha-synuclein, with a prion like pattern, leads to cause disease proliferation in the Parkinson disease under development, one of the irregular shapes of the protein islet amyloid polypeptide IAPP is readily noticeable in the beta islets of pancreas of patients affected with type 2 diabetes. Fibril of the concern protein enhances the accumulation of normal IAPP in normal presenting pancreatic tissue and succeeding development of diabetes, also associated to a prion-like module. Definitely there seems to be a prospective for communication among alpha synuclein and IAPP, in that misfolded modules of one can encourage or fasten the foundation of misfolded forms of the fact (Foltynie et al., 2006).

According to Politis et al, (2006) protein misfolding and insulin resistance leads to development of both PD and diabetes with mainly contribution of genetic susceptibility and lifestyle factors along with the involvement of the Common potential pathophysiological mechanisms like mitochondrial and endoplasmic reticulum malfunction, inflammatory response and loss of central and peripheral synapses. The Protein accumulation and prion-like proliferation have the main role in this respect. The concern thoughts were well recognized only for alpha-synuclein active in PD as a potential patho-genetic centering factor but now they have been shown as a potential cause for causing diabetes as well. The protein Islet Amyloid Polypeptide (IAPP) have ability to takes an abnormal message/signal, which then leads to change the typical protein in a prion-like manner in pancreatic islet cells concerning the DM. Fascinatingly, the strong evidence for cross-seeding between IAPP and alpha-synuclein is also confirm.

\section{Role of Mitochondria in the Association of Type 2 Diabetes and Parkinson's Disease}

The incidence rate of PD in DM patients increases with the age. Several studies in the literature indicate that PD and DM, both age-related chronic diseases, share remarkably similar pathways of mitochondrial dysfunction and suggest the association of DM with PD (Santiago \& Potashkin, 2013). To elucidate the role of type 2 DM on the risk of PD incidence, researchers performed a large cohort study of Chinese patients with and without type 2 DM using the NHIRD in Taiwan. researchers also reviewed the interaction and joint association of type 2 diabetes with comorbidities, including schizophrenia, bipolar disorder, hypertension, and hyperlipidemia, as well as flunarizine, metoclopramide use, and zolpidem on PD incidence in patients with and without type 2 DM (Sun et al., 2012).

Recent studies have revealed the importance of mitochondria as a key regulator of glucosestimulated insulin secretion in pancreatic b-cells. Increasing evidence has shown that 
mitochondrial function is closely related to various facets of diabetes, including pancreatic b-cell dysfunction, insulin resistance, obesity, and vascular complications (Wiederkehr \& Wollheim, 2006). Many diseases of mitochondrial dysfunction affect more than 1 system in the human body. They affect organs that require a lot of energy, including the heart, skeletal muscle, and brain (Kwak et al., 2010).

A study of specific gene mutations that cause PD and reinforces the relevance of oxidative stress and mitochondrial dysfunction in sporadic forms of disease in the family (Schapira, 2008). The results of the study indicate that the proteins associated with FD are either mitochondrial proteins or are associated with mitochondria, and all interact with the pathways of oxidative stress and independent radical damage (Jin et al., 2014). Although the exact cause of neuronal damage is not known, several lines of evidence suggested that mitochondrial dysfunction is one of the biochemical abnormalities described in the brains of PD patients (Fan et al., 2010; Schapira, 2008).

\section{Is Parkinson's Disease Different in Diabetics?}

Several journals reported the strong evidence of relationship b/w patients with diabetes along with developed PD show additional aggressive form of the diseases like the earlier development of cognitive impairment and postural instability. Diabetes and PD association remains as such after segregation of patients with vascular pathology and while after exclusion of patients with peripheral neuropathy in case of postural instability. Further emerging data in support of diabetes and PD association theory presented preliminary findings of a case-control research linking the DAT scans of PD patients affected with diabetes to those without diabetes patients, proposing the earlier studies showed more severe nigrostriatal degeneration (Aviles-Olmos et al., 2013).

\section{Anti-Diabetic Medications Support in Recovery of Neuroprotective Properties in Parkinson's Disease.}

The publication of two randomized PD trials of exenatide, a Glucagon-like peptide 1 (GLP-1) receptor agonist as a specific anti-diabetic medications and licensed for the treatment of type 2 diabetes proofed its efficacy in the treatment of Parkinson's disease having the ability to build GLP-1 receptor agonists having neurotrophic effects, i.e., improved performance on effects on motor and cognitive deficits of PD, and Pioglitazone (thiazolidinedione) has neuroprotective effects in a vast range. Precisely, for the treatment of PD the role of anti-diabetic agents is becoming of more importance. There is so need of knowing about the mechanisms of action specifically involved, and certainly there will be better, well-tolerated and additionally potent and high valued agents to be exposed (Ashraghi et al., 2016).

The use of a GLP-1 agonist is beneficial most for the patient having both diabetes and PD. Exenatide has been confirmed as an anti-diabetic drug to enhance glucose control, and have a tendency to cause weight loss, which is typically useful. There is already sufficient effectiveness of Exenatide on patients with diabetes and PD with capability to penetrate the blood brain barrier. However, the other GLP-1 receptor agonists have insufficient response data as in term of "to penetrate the blood brain barrier" to favor the use of Liraglutide, Semaglutide or Lixisenatide (Athauda et al., 2017).

GLP-1 agonists have also the ability to exert a neuroprotective influence by reducing vascular risk features and synaptic loss prevention. The micro and macro-vascular complications is improved 
by GLP-1 agonists via reducing cerebral ischaemia in diabetic patients. GLP-1 agonists also have main role in preventing the synapse loss by reducing the soluble $\mathrm{A} \beta$ oligomers in experimental model of Alzheimer's disease. However, it is proved from studies that the combined activation of GLP-1 and Glucose-dependent Insulinotropic Peptide (GIP) pathways have a much higher neuroprotective (neurotrophic and anti-apoptotic effects) effects (Li et al., 2010). The neuroprotective possessions are exerted by Pharmacological inhibition of dipeptidyl peptidase-4 (DPP4) via reducing inflammation, oxidative stress and apoptotic pathways, and improving mitochondrial function in parkinsonism. DPP4 inhibitors are actually anti-diabetic drugs having ability to stimulate both GLP-1 and GIP pathways. DPP4 inhibitors are capable applicants to slow PD progression, although DPP-4 inhibitors have lower capability of penetration of the blood brain barrier while compared to GLP-1 agonists (Bohnen et al., 2014). The DPP-4 inhibitors have the potency for orally administeration along with a capability of well tolerated in general, with a minor frequency of hypoglycemia and no weight addition compared to GLP-1 analogues, prominenting them well-knownr for use in combination, or as a substitute to metformin in diabetic cases. Majority of DPP-4 inhibitors are hepatically metabolized, while others are excreted by kidney (Harkavyi et al., 2008). As a result, this has offering benefits to a vast range to patients with a greater potential for their use in both renal and liver impairment. The effective and associated use of DPP-4 inhibitors with reduced incidence of PD suggested more beneficial in a clinical research conducted as a case-control study involving a large population in Sweden (Kim et al., 2009).

Exenatide expands its positive response as motor symptomatology in PD has actualized its renewed aspect on the PD-DM association on the basis of neuroprotective and regenerative effects of the drug (Fan et al., 2010). It is confirmed now that the effective response of exenatide as a tentative treatment is not disease-modifying after all. GLP-1 agonist is used as a treatment for the patients with PD, with or without DM. The outcome response of exenatide are positive, hopeful and lenience and also according to standards with respect to safety. Unforetunitely, these outcomes have capability to stimulate the biological research in the field of DM-PD associated intervention (Li et al., 2009).

Many studies revealed the relationship between DM and PD in subgroups with and without the use of medication of flunarizine, metoclopramide, and zolpidem. Moreover, DM patients who used flunarizine, metoclopramide, or zolpidem indicated larger strong degree of association than individuals with DM or each medication use alone, hence demonstrating important combined effects of DM with flunarizine, metoclopramide, and zolpidem use. DM patients with metoclopramide or zolpidem use also addressed the augmented risk of PD (Aviles-Olmos et al., 2013).

\section{Conclusions}

A great deal of present study demonstrated the precise relationships and associations between type 2 diabetes mellitus, Insulin resistance and broadly different disease, the subject of increasing interest is the Parkinson's disease among male and female independently of other potentially important confounding factors. The biological mechanisms behind the relationship of type 2 diabetes with the threat of Parkinson's disease are, however, not understood at present; therefore further mechanistic investigations are needed to validate the results. Additional findings should be acknowledged. 


\section{References}

[1] Aviles-Olmos, I., Patricia, L., Andrew, L. and Thomas, F. (2013). Parkinson's disease, insulin resistance and novel agents of neuroprotection. Brain A Journal of Neurology, 136: 374-384.

[2] Ashraghi, M. R., Pagano, G., Polychronis, S., Niccolini, F. and Politis, M. (2016). Parkinson's disease, diabetes, impairment cognitive. Recent Pat Endocr Metab Immune Drug Discov,10:11-21.

[3] Athauda, D., Maclagan, K. and Skene, S. S. (2017). Exenatide once weekly versus placebo in Parkinson's disease: a randomised, double-blind, placebo-controlled trial. Lancet, 390: 1664-1675.

[4] Barbeau, A., Giguere, R. and Hardy, J. (1961). Clinical experience with tolbutamide in Parkinson's disease. Union Med Can, 90: 147-51.

[5] Belsham, D. D., Fick, L. J., Dalvi, P. S., Centeno, M. L., Chalmers, J. A. and Lee, P. K. (2009). Ciliary neurotrophic factor recruitment of glucagon-like peptide-1mediates neurogenesis, allowing immortalization of adult murine hypothalamic neurons. FASEB, J, 23: 4256-65.

[6] Bohnen, N. I., Kotagal, V. and Muller. M. L. (2014). Diabetes mellitus is independently associated with more severe cognitive impairment in Parkinson disease. Parkinsonism Relat Disord, 20:13941398.

[7] Boyd, A. E., Lebovitz, H.E. and Feldman, J. M. (1971). Endocrine function and glucose metabolism in patients with Parkinson's disease and their alternation by L-Dopa. J Clin Endocrinol Metab, 33 : 829-37.

[8] Boulton, A. J., Vinik, A. I., Arezzo, J. C., Bril, V., Feldman, E. L. and Freeman, R. (2005). Diabetic neuropathies: a statement by the American Diabetes Association. Diabetes Care, 28: 956-62.

[9] Chalmanov, V. and Vurbanova, M. (1987). Diabetes mellitus in parkinsonism patients. Vutr Boles, 26: 68-73.

[10] Craft, S. and Watson, G.S. (2004). Insulin and neurodegenerative disease: shared and specific mechanisms. Lancet Neurol, 3:169-78.

[11] Elner, A. M. and Kandel, E.I. (1965). Studies on the Carbohydrate metabolism in Parkinsonism. (Relation of Carbohydrate metabolism disorders to the clinical picture of the disease). $\mathrm{Zh}$ Nevropatol Psikhiatr Im S S Korsakova, 65: 46-50.

[12] Fan, R., Li, X., Gu, X., Chan, J. C. and Xu, G. (2010). Exendin-4 protects pancreatic beta cells from human islet amyloid polypeptide-induced cell damage: potential involvement of AKT and mitochondria biogenesis. Diabetes Obes Metab, 12: 815-24.

[13] Harkavyi, A., Abuirmeileh, A., Lever, R., Kingsbury, A. E, Biggs, C. S. and Whitton, P.S. (2008). Glucagon-like peptide 1 receptor stimulation reverses key deficits in distinct rodent models of Parkinson's disease. J Neuro-inflammation, 5: 19.

[14] Huang, H. C., Tsai, C. H. and Muo, C. H. (2015). Risk of Parkinson's disease following zolpidem use: a retrospective, population-based cohort study. J Clin Psychiatry, 76: 104-10.

[15] Hu, G., Jousilahti, P., Bidel, S., Antikainen, R. and Tuomilehto, J. (2007). Type 2 diabetes and the risk of Parkinson's disease. Diabetes Care, 30: 842-7.

[16] International Diabetes Federation. (2015). IDF Diabetes Atlas Seventh Edition.

[17] International Diabetes Federation and World Health Organization. (2015). The diabetes declaration and strategy; a call to action and plan of action to prevent and control diabetes and related chronic diseases.

[18] Jin, H., Kanthasamy, A. and Ghosh, A, (2014). Mitochondria-targeted antioxidants for treatment of Parkinson's disease: preclinical and clinical outcomes. Biochim Biophys Acta, 1842:1282-94.

[19] Kaplan, E. L. and Meier, P. (1958). Nonparametric estimation from incomplete observations. Journal of the American Statistical Association, 53:457-81.

[20] Kim, S., Moon, M. and Park, S. (2009). Exendin-4 protects dopaminergic neurons by inhibition of microglial activation and matrix metalloproteinase-3 expression in an animal model of Parkinson's disease. J Endocrinol, 202: 431-9. 
[21] Kwak, S. H., Park, K.S. and Lee, K. U. (2010). Mitochondrial metabolism and diabetes. J Diabetes Investig,1:161-9.

[22] Lipman, I. J., Boykin, M. E. and Flora, R. E. (1974). Glucose intolerance in Parkinson's disease. J Chronic Dis, 27: 573-9.

[23] Li, Y., Tweedie, D., Mattson, M. P., Holloway, H. W. and Greig, N. H. (2010). Enhancing the GLP-1 receptor signaling pathway leads to proliferation and neuroprotection in human neuroblastoma cells. J Neurochem, 113: 1621-31.

[24] Liao, P. J., Lin, Z. Y. and Huang, J. C. (2015). The relationship between type 2 diabetic patients' early medical care-seeking consistency to the same clinician and health care system and their clinical outcomes. Medicine, 94: 554.

[25] Leibson, C. L., Rocca, W. A., Hanson, V. A., Cha, R., Kokmen, E. and O’Brien, P. C.(1997). Risk of dementia among persons with diabetes mellitus: a population-based cohort study. Am J Epidemiol, 145: 301-308.

[26] Lu, L., Fu, D. L. and Li, H. Q. (2014). Diabetes and risk of Parkinson's disease: an updated metaanalysis of case-control studies. PLoS ONE, 9: 85781.

[27] Manish, K. G., Pardeep, K., and Jugal K. (2010). Understanding survival analysis: Kaplan-Meier estimate. Int J Ayurveda Res, 1(4): 274-278. doi: 10.4103/0974-7788.76794.

[28] Morris, J. K., Vidoni, E. D. and Perea, R,D (2014). Insulin resistance and gray matter volume in neurodegenerative disease. Neuroscience, 270:139-47.

[29] Michell, A. W., Xu, Z., Fritz, D., Lewis, S. J. and Foltynie, T.(2006). Saccadic latency distributions in Parkinson's disease and the effects of Ledopa. Exp Brain Res, March 17.

[30] Noyce, Alastair, Lees, and Andrew (2017). Observations on a 2-Step Approach to Screening for Parkinson Disease. JAMA Neurology/jamaneurol, 3202.

[31] Okun, M. S., Jacob, M. D., Jones, D., Paul-Mangal, M.S., Jacob-Lafo, M.S. and Dawn-Bowers., M.S. (2016). Mood Differences Among Parkinson's Disease Patients With Mild Cognitive Impairment Ph.D.Published Online:21, https://doi.org/10.1176/appi.neuropsych.15090221.

[32] Ott, A., Stolk, R. P., van Harskamp, F., Pols, H. A., Hofman, A., Breteler, M. M. (1999). Diabetes mellitus and the risk of dementia: The Rotterdam Study. Neurology, 53: 1937-42.

[33] Peila, R., Rodriguez, B. L. and Launer, L. J. (2002). Type 2 diabetes, APOE gene, and the risk for dementia and related pathologies: The Honolulu-Asia Aging Study. Diabetes, 51: 1256-62.

[34] Politis, Marios \& Reza Ashraghi, Mohammad \& Pagano, Gennaro \& Polychronis, Sotirios \& Niccolini, and Flavia (2016). Parkinson's Disease, Diabetes and Cognitive Impairment. Recent patents on endocrine, metabolic \& immune drug discovery. 10. 10.2174/1872160630191119.

[35] Pressley, J. C., Louis, E. D., Tang, M. X., Cote, L., Cohen, P. D. and Glied, S. (2003). The impact of comorbid disease and injuries on resource use and expenditures in Parkinsonism. Neurology, 60: 87-93.

[36] Saguil, A. and Lax, J. W. (2014). Acute migraine treatment in emergency settings. Am Fam Physician, 89:742-4.

[37] Santiago, J. A. and Potashkin, J. A. (2013). Shared dysregulated pathways lead to Parkinson's disease and diabetes. Trends Mol Med,19:176-86.

[38] Sandy, k. R. (1993). The relationship between diabetes mellitus and Parkinson's disease. Int J Neurosci, 69: 125-30.

[39] Schapira, A. H. (2008). Mitochondria in the aetiology and pathogenesis of Parkinson's disease. Lancet Neurol, 7: 97-109.

[40] Schwab, R. S. (1960). Progression and prognosis in Parkinson's disease. J Nerv Ment Dis, 130: 556-66.

[41] Schapira, A. H. (2008). Mitochondria in the aetiology and pathogenesis of Parkinson's disease. Lancet Neurol, 7:97-109. 
[42] Sun, Y., Chang, Y. H. and Chen, H. F.(2012). Risk of Parkinson disease onset in patients with diabetes: A 9-year population-based cohort study with age and sex stratifications. Diabetes Care, 35:1047-9.

[43] Szendroedi, J., Phielix, E. and Roden, M. (2012) The role of mitochondria in insulin resistance and type 2 diabetes mellitus. Nat Rev Endocrinol, 8:92-103.

[44] Tuomilehto, J., Rastenyte, D., Jousilahti, P., Sarti, C. and Vartiainen, E. (1996). Diabetes mellitus as a risk factor for death from stroke. Prospective study of the middle-aged Finnish population. Stroke, 27: 210-5.

[45] Van-Woert, M. H. and Mueller, P. S. (1971). Glucose, insulin, and free fatty acid metabolism in Parkinson's disease treated with levodopa. Clin Pharmacol Ther, 12: 360-7.

[46] Warner, T.T., Casper, C. J., Taanman, J. W. and Nathwani, A.C. (2012). Generation of iPS-derived dopaminergic neurons as an in-vitro model of rapid-onset dystonia parkinsonism. Hum Gene Therapy, 22, A42-A43.

[47] Wahlqvist, M. L., Lee, M. S. and Hsu, C.C. (2012). Metformin-inclusive sulfonylureatherapy reduces the risk of Parkinson's disease occurring with type 2 diabetes in a Taiwanese population cohort. Parkinsonism Relat Disord,18:753-8.

[48] Wiederkehr, A. and Wollheim, C. B. (2006). Minireview: implication of mitochondria in insulin secretion and action. Endocrinology, 147:2643-9.

[49] Yue, X., Li, H., Yan, H., Zhang, P., Chang, L. and Li, T. (2016). Risk of Parkinson disease in diabetes mellitus: an updated meta-analysis of population-based cohort studies. Medicine, 95: 3549.

[50] Yu-Wan, Y., Teng-Fu, H., Chia-Ing, L., Chiu-Shong, L., Wen-Yuan, L. and Jen-Huai, C. (2008). Increased risk of Parkinson disease with diabetes mellitus in a population-based study Journal of Medicine, 96: 1-10.

[51] Zimmet, P., Alberti, K. G., Shaw, J. (2001). Global and societal implications of the diabetes epidemic. Nature, 414: 782-7

\footnotetext{
*Corresponding author.

E-mail address: hur_2085qta@ yahoo.com
} 